\title{
Changes in resting-state connectivity in pediatric temporal lobe epilepsy
}

\author{
Fabio Grassia, MD, ${ }^{1}$ Andrew V. Poliakov, PhD, ${ }^{2,4}$ Sandra L. Poliachik, PhD, ${ }^{2,4}$ Kaitlyn Casimo, $^{2}$ \\ Seth D. Friedman, PhD, ${ }^{2}$ Hillary Shurtleff, PhD, ABPP, ${ }^{3}$ Carlo Giussani, MD, PhD, ${ }^{1}$ \\ Edward J. Novotny Jr., MD, ${ }^{3}$ Jeffrey G. Ojemann, MD, ${ }^{4,5}$ and Jason S. Hauptman, MD, PhD ${ }^{4,5}$ \\ 'Department of Neurological Surgery, University of Milano-Bicocca, San Gerardo Hospital, Monza, Italy; Departments of \\ ${ }^{2}$ Radiology and ${ }^{3}$ Neurology, Seattle Children's Hospital; ${ }^{4}$ Department of Neurological Surgery, University of Washington; and \\ ${ }^{5}$ Department of Neurosurgery, Seattle Children's Hospital, Seattle, Washington
}

\begin{abstract}
OBJECTIVE Functional connectivity magnetic resonance imaging (fcMRI) is a form of fMRI that allows for analysis of blood oxygen level-dependent signal changes within a task-free, resting paradigm. This technique has been shown to have efficacy in evaluating network connectivity changes with epilepsy. Presurgical data from patients with unilateral temporal lobe epilepsy were evaluated using the fcMRI technique to define connectivity changes within and between the diseased and healthy temporal lobes using a within-subjects design.
\end{abstract}

METHODS Using presurgical fcMRI data from pediatric patients with unilateral temporal lobe epilepsy, the authors performed seed-based analyses within the diseased and healthy temporal lobes. Connectivity within and between temporal lobe seeds was measured and compared.

RESULTS In the cohort studied, local ipsilateral temporal lobe connectivity was significantly increased on the diseased side compared to the healthy temporal lobe. Connectivity of the diseased side to the healthy side, on the other hand, was significantly reduced when compared to connectivity of the healthy side to the diseased temporal lobe. A statistically significant regression was observed when comparing the changes in local ipsilateral temporal lobe connectivity to the changes in inter-temporal lobe connectivity. A statistically significant difference was also noted in ipsilateral connectivity changes between patients with and those without mesial temporal sclerosis.

CONCLUSIONS Using fCMRI, significant changes in ipsilateral temporal lobe and inter-temporal lobe connectivity can be appreciated in unilateral temporal lobe epilepsy. Furthermore, fcMRI may have a role in the presurgical evaluation of patients with intractable temporal lobe epilepsy.

https://thejns.org/doi/abs/10.3171/2018.3.PEDS17701

KEYWORDS temporal lobe epilepsy; functional connectivity MRI; surgical technique

$\mathrm{F}$ UNCTIONAL connectivity MRI (fcMRI) - a method of analyzing blood oxygen level-dependent (BOLD) signal in a task-free, resting-state paradigm-can provide insight into regional connectivity in both the intact and diseased brain. In healthy controls, fcMRI reveals compartmentalization of neuronal networks into specific functional systems, such as sensorimotor, visual, auditory, dorsal attention, and default mode network (DMN)., While task-based fMRI is routinely used as part of the clinical presurgical evaluation of pediatric epilepsy patients, fcMRI is an emerging addition to the imaging armamentarium. Several studies have addressed the question of fcMRI connectivity in epilepsy, ${ }^{5}$ including in temporal lobe epilepsy (TLE) cases..$^{10}$ These studies, summarized in Mankinen et al. ${ }^{10}$ compared connectivity in patients versus healthy controls, thus using a between-subjects design. Although these studies found connectivity abnormalities in a number of networks at a population level, they did not address lateralizing changes in resting-state fcMRI in the individual patient.

Constable et al. recently reviewed the potential use and challenges of functional connectivity mapping in intractable epilepsy. ${ }^{6}$ The most common method used in fcMRI processing is seed-based analysis. Seed-based analysis es-

ABBREVIATIONS BOLD = blood oxygen level-dependent; DMN = default mode network; fcMRI = functional connectivity MRI; MNI = Montreal Neurological Institute; MTS $=$ mesial temporal sclerosis; $\mathrm{ROI}=$ region of interest; TLE = temporal lobe epilepsy.

SUBMITTED December 11, 2017. ACCEPTED March 29, 2018.

INCLUDE WHEN CITING Published online June 22, 2018; DOI: 10.3171/2018.3.PEDS17701. 
TABLE 1. Summary of demographics and MRI findings

\begin{tabular}{|c|c|c|c|c|c|c|c|}
\hline $\begin{array}{l}\mathrm{Pt} \\
\text { No. }\end{array}$ & $\begin{array}{c}\text { Age at Op } \\
\text { (yrs) }\end{array}$ & Sex & $\begin{array}{c}\text { Sz } \\
\text { Laterality }\end{array}$ & MRI Finding & $\begin{array}{c}\text { Sedation } \\
\text { During MRI }\end{array}$ & Surgery & Postop Outcome \\
\hline 1 & 17.2 & $\mathrm{~F}$ & $\mathrm{Rt}$ & Negative & No & Rt temporal lobectomy & 1 yr: Engel I \\
\hline 2 & 8.4 & M & $\mathrm{Lt}$ & $\begin{array}{l}\text { Dysplasia; It mesial } \\
\text { temporal lobe }\end{array}$ & No & Rt temporal lobectomy & $\begin{array}{l}6 \text { mos: Engel I, followed by recurrence; } \\
\text { now Engel IV at } 1 \mathrm{yr}\end{array}$ \\
\hline 3 & 17.5 & $\mathrm{~F}$ & Rt & Negative & No & Rt temporal lobectomy & $\begin{array}{l}6 \text { mos: Engel I, followed by recurrence; } \\
\text { now Engel III at } 1 \text { yr }\end{array}$ \\
\hline 4 & 13.1 & M & $\mathrm{Lt}$ & MTS & No & Lt temporal lobectomy & 2 yrs: Engel I \\
\hline 5 & 6.3 & $\mathrm{~F}$ & $\mathrm{Rt}$ & Negative & Yes & Rt temporal lobectomy & 2.5 yrs: Engel I \\
\hline 6 & 17.3 & M & $\mathrm{Lt}$ & MTS & No & Lt temporal LITT & 3 yrs: Engel II \\
\hline 7 & 15.6 & M & $\mathrm{Lt}$ & MTS & No & Lt temporal LITT & 3 yrs: Engel II \\
\hline 8 & 13.4 & M & $\mathrm{Lt}$ & Negative & No & Lt temporal lobectomy & 3.5 yrs: Engel III \\
\hline 9 & 17.8 & $\mathrm{~F}$ & $\mathrm{Lt}$ & Negative & No & Lt temporal LITT & 2 yrs: Engel III \\
\hline 10 & 5.5 & M & $\mathrm{Lt}$ & MTS & Yes & Lt temporal lobectomy & 1.5 yrs: Engel I \\
\hline 11 & 11.3 & M & Lt & MTS & No & Lt temporal LITT & 4 mos, Engel I \\
\hline 12 & 19.4 & $\mathrm{~F}$ & $\mathrm{Lt}$ & MTS & No & Lt temporal lobectomy & 6 mos, Engel II \\
\hline 13 & 9.1 & $M$ & $\mathrm{Lt}$ & MTS & Yes & Lt temporal lobectomy & $\begin{array}{l}1 \text { yr: Engel I, followed by recurrence; } \\
\text { now Engel III at } 4.5 \text { yrs }\end{array}$ \\
\hline 14 & 5.0 & M & $\mathrm{Lt}$ & MTS & Yes & Lt temporal lobectomy & 5 yrs: Engel I \\
\hline 15 & 0.7 & $\mathrm{~F}$ & $\mathrm{Lt}$ & $\begin{array}{l}\text { Dysplasia; It parietal \& } \\
\text { temporal lobes }\end{array}$ & Yes & $\begin{array}{l}\text { Lt temporoparietal } \\
\text { resection }\end{array}$ & $\begin{array}{l}1 \text { yr: Engel; underwent subsequent } \\
\text { hemispherectomy; now Engel I }\end{array}$ \\
\hline 16 & 7.0 & $\mathrm{~F}$ & Rt & Tuber; rt temporal & Yes & Rt resection of tuber & 5.5 yrs: Engel I \\
\hline 17 & 3.8 & M & $\mathrm{Lt}$ & Tumor; It temporal tip & Yes & Lt resection of tumor & 6 yrs: Engel I \\
\hline 18 & 17.2 & M & $\mathrm{Lt}$ & Negative & Yes & Lt temporal lobectomy & 5.5 yrs: Engel I \\
\hline
\end{tabular}

LITT = laser interstitial thermal therapy; $\mathrm{Pt}=$ patient; $\mathrm{Sz}=$ seizure.

timates changes in seed or region of interest (ROI) BOLD signal to whole-brain connectivity maps. In epilepsy, this approach has been used to examine changes in language networks before and after anterior temporal lobe resection. ${ }^{3}$ Pereira et al. examined connectivity between the left and right hippocampi in mesial temporal sclerosis (MTS) patients. ${ }^{14}$ They found changes in functional connectivity between hippocampi in patients with unilateral disease. A similar study using each of the left and right hippocampi as seeds for a seed-to-whole-brain analysis was performed, showing changes in resting-state functional connectivity between the hippocampi and thalamic nuclei. ${ }^{12}$

We hypothesized that the temporal pole might be a productive ROI for studying intra- and interlobar connectivity in individual TLE patients. Furthermore, comparing fcMRI patterns in the affected lobe versus the unaffected lobe might provide additional information about the extent of the epileptogenic network and changes in connectivity that occur in intractable epilepsy. We retrospectively applied this technique to patients with TLE. We studied the influence of these disease processes on resting-state fcMRI patterns in the temporal lobes as well as the interhemispheric connectivity. We then compared the connectivity measures in the affected versus unaffected temporal lobes.

\section{Methods}

Twenty-nine patients with drug-resistant TLE being considered for epilepsy surgery were identified from July
2012 to May 2017 at Seattle Children's Hospital. Only subjects noted to have unilateral temporal lobe disease were included. These patients were studied using fcMRI as part of a comprehensive phase 1 evaluation that included 3-T structural MRI, FDG-PET, video EEG monitoring with ictal recordings, and neuropsychological and developmental testing. Exclusion criteria were excessive motion during fcMRI (making processing suboptimal), metal artifact (e.g., dental appliances), or gross anatomical asymmetry in brain appearance that prevented reproducible seed-based analysis using temporal lobe seeds in standard Montreal Neurological Institute (MNI) space. Eighteen patients (7 female [mean age $11.4 \pm 5.6$ years], including 8 sedated patients [mean age $6.8 \pm 4.5$ years]) met inclusion and exclusion criteria (see Table 1 for a brief listing of clinical and demographic parameters). This retrospective study was approved by the IRB at Seattle Children's Hospital.

Imaging was performed on a Siemens (Erlangen) 3-T scanner (MAGNETOM Trio; 12-, 32-, or 64-channel head coil) with the following protocol: echo-planar imaging, blood oxygen level-dependent (BOLD) sequence, TR 2 sec, TE 27 msec, flip angle $78^{\circ}$, 34 slices, FOV $256 \times 256$ $\mathrm{mm}$, and voxel size $4 \times 4 \times 4 \mathrm{~mm}$. Resting-state fcMRI scans were obtained with patients either awake or anesthetized with propofol, depending on whether they could tolerate the study without anesthesia. Conscious patients (10 out of 18) were instructed to relax and rest while keeping their eyes open. The fcMRI study was incorporated into either the clinical anatomical MRI or the tasked-based 
fMRI studies that were part of the patients' routine presurgical evaluation.

Data analysis was performed using 1000 Functional Connectomes Project (http://www.nitrc.org/projects/ fcon_1000/) scripts based on Analysis of Functional NeuroImages (AFNI, http://afni.nimh.nih.gov/afni) and Functional Magnetic Resonance Imaging of the Brain Software Library (FSL, http://www.fmrib.ox.ac.uk/fsl) software packages. An earlier publication describes our data acquisition and analysis in more detail. ${ }^{9}$ In brief, data were temporarily filtered $(0.005-0.1 \mathrm{~Hz})$ and spatially smoothed (6$\mathrm{mm}$ full width at half maximum Gaussian blur). Sources of spurious variance along with their temporal derivatives were removed from the data through linear regression: 1) 6 parameters obtained by rigid-body correction of head motion; 2) the whole-brain signal, averaged over a fixed region in atlas space; 3) signal from a ventricular region; and 4) signal from a region centered in the white matter. Correlation maps were produced by extracting the BOLD time course from a seed region, then computing the correlation coefficient between that time course and the time course from all other brain voxels. The correlation maps were converted to normal distribution by Fisher's z-transform. These values were converted to $\mathrm{z}$-scores by dividing the square root of the variance, calculated as $1 / \sqrt{ }(n-3)$, where $\mathrm{n}$ represents the degrees of freedom of the measurement. The degrees of freedom were calculated according to Bartlett's theory using the BOLD time series-lagged autocovariance function averaged over multiple ROIs. Finally, z-score maps were thresholded at a $\mathrm{z}$ value of 3.0. We routinely analyzed the resting-state data for connectivity with the following seeds (MNI coordinates): somatomotor $(\mathrm{SM} ; \pm 39,-27,51)$; visual $(\mathrm{V} 1 ; \pm 18,-72,12)$; auditory $(\mathrm{A} 1 ; \pm 60,-12,0)$; and $\mathrm{DMN} /$ posterior cingulate cortex $(\mathrm{PCC} ; \pm 6,-48,39) .{ }^{16}$

For the purposes of this study, we analyzed the connectivity of the A1 seed located in the anterior temporal lobe to the whole temporal lobe regions, as defined by the MNI structural atlas in FSL software (the temporal lobe masks were symmetrized). As a measure of connectivity, we chose the volume of all voxels that exceeded the $\mathrm{z}$ value threshold. As a control, the same measurement was performed for the V1 seed with respect to the occipital lobe.

\section{Results}

Compared to healthy control subjects (Fig. 1A and B), studies in epileptic patients demonstrated a variety of connectivity patterns. These patterns ranged from reduced connectivity (Fig. 2A), to complete lack of connectivity (Fig. 2B and C), to a near-normal pattern (Fig. 2D). In some of the cases (e.g., Fig. 2B), connectivity in the ipsilateral hemisphere appeared to show atypical pattern and spread compared to both the unaffected hemisphere and expected normal connectivity pattern. In other cases (Fig. 2D), connectivity seemed to be quite similar to that expected in normal patterns, possibly indicating that the disease process had not yet produced network changes.

We did not attempt to quantitatively compare findings in patients with those in normal controls, but rather we compared the connectivity of the affected temporal lobe
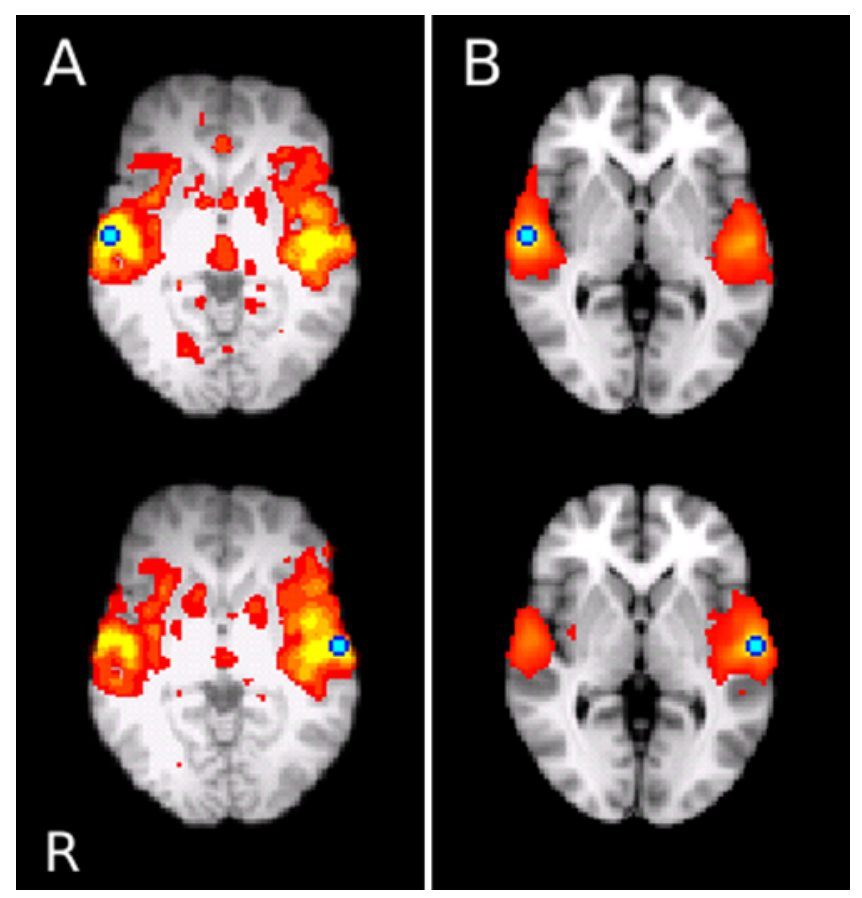

FIG. 1. Functional connectivity of temporal lobe seeds in healthy controls. Connectivity patterns of the left (upper) and the right (lower) temporal seeds with $\mathrm{MNI}$ coordinates $( \pm 60,-12,0)$ in control subjects. A: Images in 12-year-old, awake control subject showing fairly typical connectivity for normal subjects. B: Average connectivity for images obtained in 10 healthy teenage control subjects overlaid onto atlas brain image (MNI 152 T1 volume). Both demonstrate strong connectivity between the seeds and both ipsilateral and contralateral anterior temporal poles.

with the connectivity of the unaffected lobe within a given patient. One common finding was that connectivity from the A1 seed on the affected side to the opposite temporal lobe was reduced compared to the connectivity from the symmetric A1 seed on the unaffected side to the diseased temporal lobe. We computed seed-to-temporal lobe connectivity values as volume of connected area and found that connectivity on the affected side tended to be greater on average by $20 \%$ (affected side mean $229 \pm 135 \mathrm{~mm}^{3}$, unaffected side mean $274 \pm 127 \mathrm{~mm}^{3}$ ). A paired t-test showed that reduction in connectivity was statistically significant $(p=0.029)$. Also, the connectivity of the seed on the affected side to the ipsilateral temporal lobe tended to be more widespread than the unaffected side by, on average, 12\% (affected side mean $455 \pm 203 \mathrm{~mm}^{3}$, unaffected side mean $385 \pm 91 \mathrm{~mm}^{3}$ ). Again, a paired t-test showed this increase in connectivity to be statistically significant $(p=0.046)$. We then performed linear regression, comparing the changes in connectivity from the seed to the contralateral temporal lobe with the changes from the seed to the ipsilateral temporal lobe per patient. Linear regression modeling with a 0 intercept showed that connectivity was inversely related across patients (Fig. 3; $\mathrm{I}=-0.3039 \mathrm{C}$, where $\mathrm{I}$ is ipsilateral and $\mathrm{C}$ is contralateral connectivity differences; $p=0.015$; correlation coefficient -0.46 ). We then performed the same analysis for visual V1 seed with 

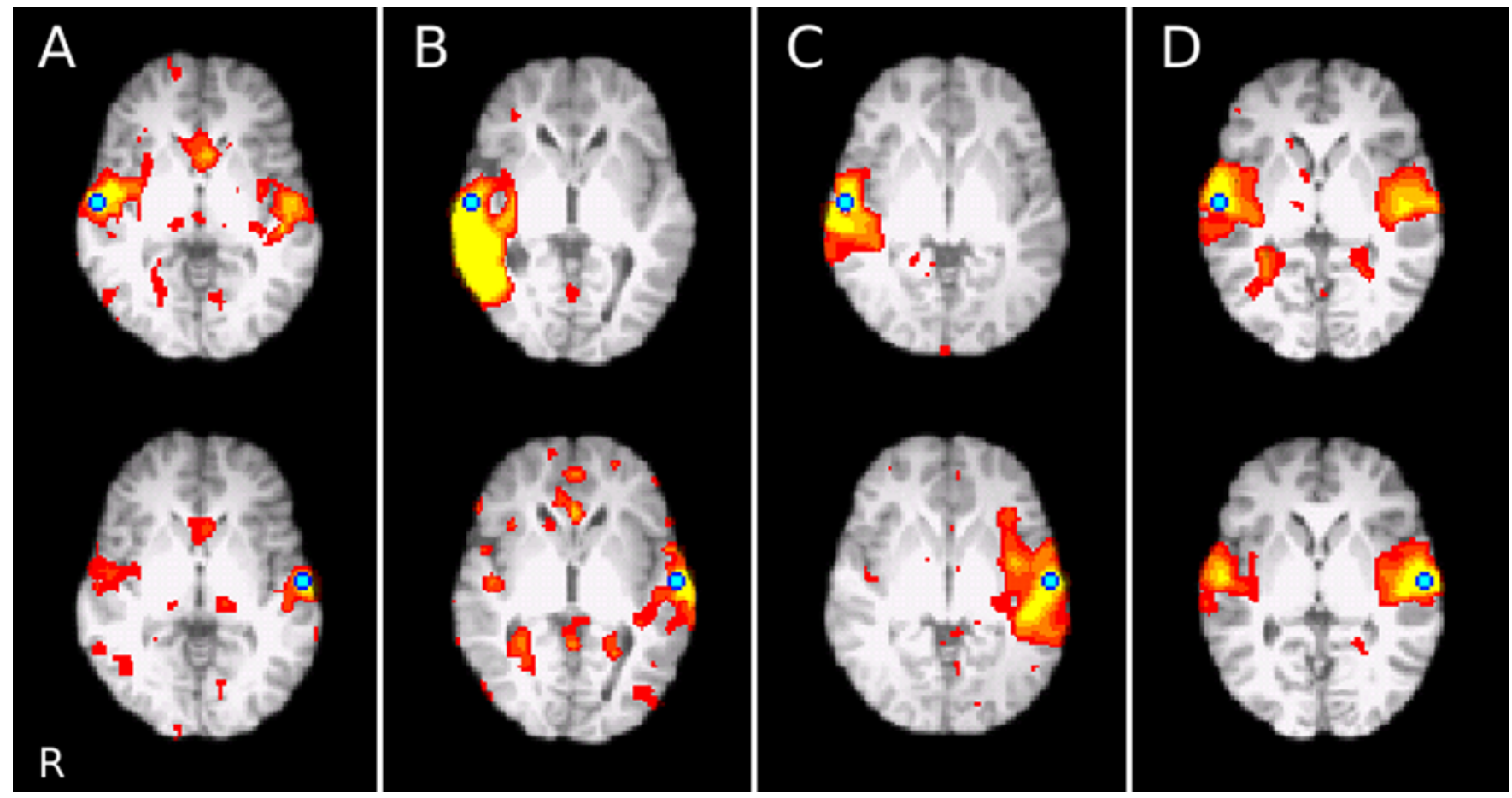

FIG. 2. Functional connectivity of temporal lobe seeds in patients with TLE. A: Patient 6: a 17-year-old boy with MTS and left TLE. Images show reduced connectivity between the seed in the left (affected) lobe and the contralateral temporal lobe, compared to the seed in the unaffected right lobe. B: Patient 16: a 7-year-old girl with tuberous sclerosis and right TLE. Images demonstrate lack of detectable connectivity between the seed on the right (affected) side and the contralateral temporal lobe. In addition, connectivity of this seed and the ipsilateral lobe appears to be stronger and more widespread than that of the unaffected left lobe. C: Patient 18: a 17-year-old boy with MRI-negative left TLE showed lack of detectable connectivity between the seed on the left (affected) side and the contralateral temporal lobe, and vice versa. D: Patient 1: a 17-year-old girl with MRI-negative right TLE. Images show an apparently normal connectivity pattern despite disease within the right temporal lobe.

respect to occipital lobes as a control. No trends or statistically significant changes were detected.

As an additional analysis, we looked for relationships between changes in connectivity and etiology. We found that in patients with radiological MTS, change in ipsilateral connectivity was significantly different compared with that seen in patients without MTS (Fig. 4; $-344 \pm 295 \mathrm{~mm}^{3}$ vs $651 \pm 501 \mathrm{~mm}^{3} ; \mathrm{p}=0.001$ ).

\section{Discussion}

Resting-state functional connectivity patterns have been well studied in healthy subjects. ${ }^{716}$ In patients with refractory epilepsy undergoing fcMRI as part of the presurgical evaluation, the data are still somewhat limited but growing. ${ }^{213}$ Disruption of networks has been described in temporal lobe epilepsy, ${ }^{2,17}$ including in children, ${ }^{5}$ and increased local connectivity has been found to correlate with abnormal EEG foci. ${ }^{10}$ Increased connectivity between hemispheres has also been reported in absence epilepsy. ${ }^{6}$ The vast majority of reports published to date focus on comparing the TLE patient group with healthy controls. Although these studies provide valuable information about the nature of TLE, it is equally important to define connectivity changes within subjects by comparing patterns according to laterality. This allows fcMRI resting-state data signals to be compared under identical conditions. Bonelli et al. ${ }^{3}$ analyzed fcMRI data of 4 individual nonlesional TLE patients and demonstrated that measures such as regional homogeneity and interregional connectivity show a strong trend toward being reduced in the epileptic focus. Low regional homogeneity values suggest high local connectivity, as it is a measure of signal similarity for neighboring voxels. While we did not attempt to correlate fcMRI to epileptic focus in this particular study, increased local connectivity (and thus, reduced regional homogeneity) near epileptic foci would be consistent with our findings here.

Several studies have suggested that fcMRI may serve as an adjunct presurgical tool, facilitating the identification of the seizure focus in focal epilepsy. ${ }^{5,10} \mathrm{We}$ assessed the connectivity of the atlas-based seed placed in the tip of the temporal lobe. We hypothesized that the epileptic process might be altering normal intra- and interlobar connectivity patterns. As expected, the patient population is diverse, and thus we observed expected substantial variability in connectivity patterns and measurements within the cohort. That said, we found three statistically significant changes in connectivity maps within our population: a relative reduction in connectivity between the seed in the diseased temporal lobe and the healthy temporal lobe compared to the converse; a relative increase in connectivity within the diseased temporal lobe compared to the 


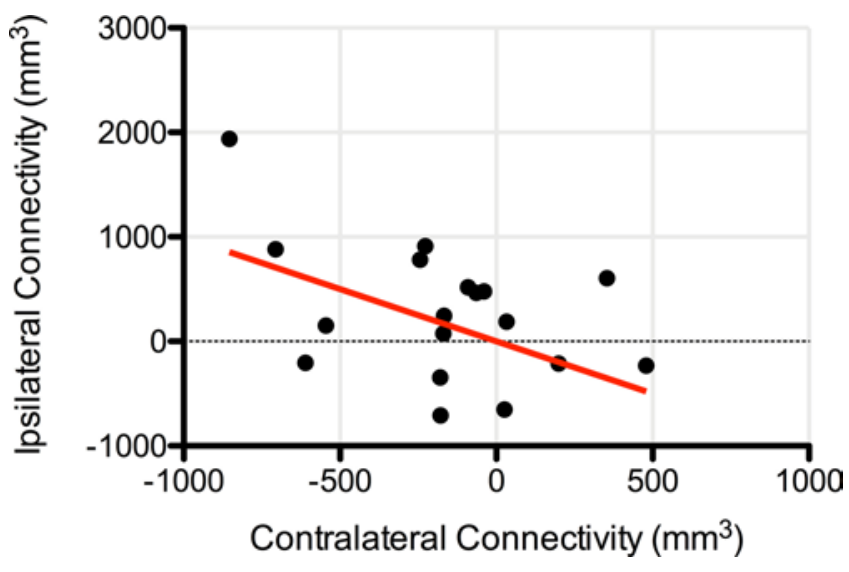

FIG. 3. Inverse relations between the increment in the connectivity within the affected lobe and the reduction of connectivity to the unaffected side. Connectivity was measured as a volume $\left(\mathrm{mm}^{3}\right)$ of all voxels that exceeded the statistical threshold.

healthy temporal lobe; and an inverse relationship between these two characteristics. Interestingly, this trend was not observed in the occipital lobe within the cohort. In all, these findings suggest a shift in the balance of connectivity on the side of presumed epileptogenicity (the diseased temporal lobe): more local connections and fewer contralateral connections. Given that our regression model was statistically significant, we could in theory predict the degree of contralateral connectivity reduction based on the degree of local connectivity increase. To our knowledge, this is the first time this relationship has been described in the literature. Additionally, the significant difference in local connectivity in MTS versus non-MTS patients is a curious observation that may speak to a different network shift in this particular disease state. Our data suggest that this change in connectivity may be a different process than that in the non-MTS cohorts. Interestingly, other groups have noted reductions in intrahemispheric functional connectivity in MTS, albeit in other circuits. ${ }^{15}$ Others have also previously suggested that there may be connectivity differences between cortical dysplasia and MTS, which would be consistent with our findings. ${ }^{4}$

As we begin to understand this phenomenon further, it would be interesting to see if an additional covariate in the regression model would be the severity of underlying epilepsy, as well as the duration of the disease. Haneef et al. looked at changes in the homogeneity of connectivity maps as a function of TLE duration and suggested that the diversity of connectivity diminishes as the duration of epilepsy increases. ${ }^{8}$ One possible hypothesis would be that the greater the severity and/or duration of the epilepsy, the greater is the increase in local connectivity within the diseased temporal lobe and the greater is the reduction in contralateral temporal lobe connectivity to the healthy temporal lobe from the diseased side. An explanation for these findings may be alteration and deterioration of networks due either to the epilepsy disease process or to ongoing subclinical epileptiform activity during fcMRI data acquisition. It is also possible that these findings represent structural network differences within the diseased tempo-

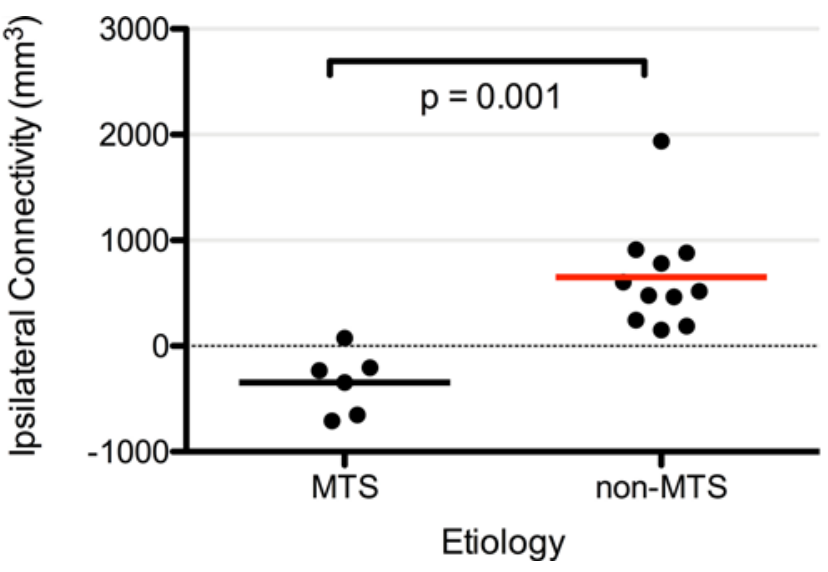

FIG. 4. Significant difference between the increment in the connectivity within the affected lobe in patients with radiological MTS compared to that in those patients with other etiologies (dysplasia, tumor, or MRInegative disease). Connectivity was measured as a volume $\left(\mathrm{mm}^{3}\right)$ of all voxels that exceeded the statistical threshold.

ral lobe, potentially developmental in nature, that could be contributing to the seizure disorder itself. Given the differences in connectivity maps across our cohort, there is a strong suggestion that we are looking at a dynamic, heterogeneous process. Regardless, because fcMRI connectivity only reflects correlation coefficient, causality is uncertain. It is possible increased BOLD signal in the temporal lobe ipsilateral to the disease simply represents homogeneity, and this may be due to neuronal suppression during the interictal period, thus not necessarily indicating that the neurons are "more connected." Clearly, this observation is an epiphenomenon, and while the mechanistic implications are unclear, there could be utility in studying it further as part of the clinical presurgical workup and potentially also in response to temporal lobe connectivity postsurgically as it relates to seizure-free outcomes and neuropsychological changes.

Our working hypothesis is that the increase of connectivity within the epileptic lobe reflects BOLD signal homogeneity secondary to ictal and interictal changes modifying networks around the focus. This could also explain the reduced connectivity between the diseased temporal lobe and the contralateral lobe, as one would expect the homogeneity of local BOLD maps to vary from that of maps of the unaffected temporal lobe. The inverse relationship that we noted with regard to connectivity of the diseased temporal lobe to the healthy temporal lobe could potentially be explained in light of network injury due to ongoing seizures, reorganization favoring local connectivity/BOLD homogeneity, and/or developmental digression of temporotemporal networks, leading to TLE as a secondary phenomenon.

We hope that future endeavors will help further our understanding of this phenomenon and help us apply it to the presurgical evaluation of patients with intractable epilepsy, in either the realm of surgical approach or postoperative prognostication. Morgan et al. used a prediction model of seizure freedom based on connectivity within a defined, proposed seizure propagation network, ${ }^{11}$ and similarly we 
would like evaluate our measurements prognostically in the future. There could also be utility in applying this imaging approach in patients with MRI-negative TLE, with the hope that the changes in connectivity noted here might correlate with diseased temporal lobes lacking the ultrastructural changes necessary to be visualized on standard anatomical imaging modalities.

Despite the statistical significance achieved, this is a limited preliminary study with a small and diverse patient cohort. In order to make resting-state connectivity studies relevant to clinical applications in epilepsy, methods of data acquisition and processing need to be improved. In particular, motion artifact remains a limiting factor in achieving robust and standardized connectivity patterns and measurements that are reproducible both between and within subjects. One potential solution would be to develop and implement fragmented analysis of resting-state data that would effectively exclude time points affected by motion. Evolving techniques of MRI data acquisition, including multiband and other techniques that greatly increase time resolution, may contribute to improvement in resting-state fMRI clinical applications.

\section{Conclusions}

Using fcMRI, significant changes in ipsilateral temporal lobe and inter-temporal lobe connectivity can be appreciated in unilateral temporal lobe epilepsy. These may be representative of network evolution, leading to BOLD signal alterations from ictal and interictal activity. The specific ipsilateral network changes in MTS observed in this cohort corroborate previous observations regarding differences in connectivity across pathologies. Furthermore, fcMRI may have a role in the presurgical evaluation of patients with intractable temporal lobe epilepsy. Much work still needs to be done with regard to refinement of acquisition and analysis of data. Also, larger cohorts will be needed to achieve a greater granularity as it pertains to how connectivity changes with specific pathologies, how it varies over the course of the epilepsy, and how to use it to assist lateralization/localization.

\section{References}

1. Beckmann CF, DeLuca M, Devlin JT, Smith SM: Investigations into resting-state connectivity using independent component analysis. Philos Trans R Soc Lond B Biol Sci 360:1001-1013, 2005

2. Bettus G, Bartolomei F, Confort-Gouny S, Guedj E, Chauvel P, Cozzone PJ, et al: Role of resting state functional connectivity MRI in presurgical investigation of mesial temporal lobe epilepsy. J Neurol Neurosurg Psychiatry 81:11471154,2010

3. Bonelli SB, Thompson PJ, Yogarajah M, Vollmar C, Powell $\mathrm{RH}$, Symms MR, et al: Imaging language networks before and after anterior temporal lobe resection: results of a longitudinal fMRI study. Epilepsia 53:639-650, 2012

4. Caciagli L, Bernhardt BC, Hong SJ, Bernasconi A, Bernasconi $\mathrm{N}$ : Functional network alterations and their structural substrate in drug-resistant epilepsy. Front Neurosci 8:411, 2014

5. Centeno M, Carmichael DW: Network connectivity in epilepsy: resting state fMRI and EEG-fMRI contributions. Front Neurol 5:93, 2014

6. Constable RT, Scheinost D, Finn ES, Shen X, Hampson M,
Winstanley FS, et al: Potential use and challenges of functional connectivity mapping in intractable epilepsy. Front Neurol 4:39, 2013

7. Fox MD, Snyder AZ, Vincent JL, Corbetta M, Van Essen DC, Raichle ME: The human brain is intrinsically organized into dynamic, anticorrelated functional networks. Proc Natl Acad Sci U S A 102:9673-9678, 2005

8. Haneef Z, Chiang S, Yeh HJ, Engel J Jr, Stern JM: Functional connectivity homogeneity correlates with duration of temporal lobe epilepsy. Epilepsy Behav 46:227-233, 2015

9. Khanna PC, Poliakov AV, Ishak GE, Poliachik SL, Friedman SD, Saneto RP, et al: Preserved interhemispheric functional connectivity in a case of corpus callosum agenesis. Neuroradiology 54:177-179, 2012

10. Mankinen K, Jalovaara P, Paakki JJ, Harila M, Rytky S, Tervonen $\mathrm{O}$, et al: Connectivity disruptions in resting-state functional brain networks in children with temporal lobe epilepsy. Epilepsy Res 100:168-178, 2012

11. Morgan VL, Englot DJ, Rogers BP, Landman BA, Cakir A, Abou-Khalil BW, et al: Magnetic resonance imaging connectivity for the prediction of seizure outcome in temporal lobe epilepsy. Epilepsia 58:1251-1260, 2017

12. Morgan VL, Sonmezturk HH, Gore JC, Abou-Khalil B: Lateralization of temporal lobe epilepsy using resting functional magnetic resonance imaging connectivity of hippocampal networks. Epilepsia 53:1628-1635, 2012

13. Negishi M, Martuzzi R, Novotny EJ, Spencer DD, Constable RT: Functional MRI connectivity as a predictor of the surgical outcome of epilepsy. Epilepsia 52:1733-1740, 2011

14. Pereira FR, Alessio A, Sercheli MS, Pedro T, Bilevicius E, Rondina JM, et al: Asymmetrical hippocampal connectivity in mesial temporal lobe epilepsy: evidence from resting state fMRI. BMC Neurosci 11:66, 2010

15. Tang Y, Xia W, Yu X, Zhou B, Luo C, Huang X, et al: Shortterm cerebral activity alterations after surgery in patients with unilateral mesial temporal lobe epilepsy associated with hippocampal sclerosis: a longitudinal resting-state fMRI study. Seizure 46:43-49, 2017

16. Van Dijk KR, Hedden T, Venkataraman A, Evans KC, Lazar SW, Buckner RL: Intrinsic functional connectivity as a tool for human connectomics: theory, properties, and optimization. J Neurophysiol 103:297-321, 2010

17. Waites AB, Briellmann RS, Saling MM, Abbott DF, Jackson GD: Functional connectivity networks are disrupted in left temporal lobe epilepsy. Ann Neurol 59:335-343, 2006

\section{Disclosures}

The authors report no conflict of interest concerning the materials or methods used in this study or the findings specified in this paper.

\section{Author Contributions}

Conception and design: Grassia, Giussani, Ojemann. Acquisition of data: Grassia, Poliakov, Poliachik, Casimo, Friedman, Novotny. Analysis and interpretation of data: Hauptman, Grassia, Poliakov, Poliachik, Casimo, Friedman. Drafting the article: Hauptman, Grassia, Poliakov, Shurtleff. Critically revising the article: Hauptman, Shurtleff, Giussani, Novotny, Ojemann. Reviewed submitted version of manuscript: Hauptman, Grassia, Poliakov, Poliachik, Friedman, Shurtleff, Giussani, Novotny, Ojemann. Approved the final version of the manuscript on behalf of all authors: Hauptman. Statistical analysis: Grassia, Poliakov. Study supervision: Hauptman.

\section{Correspondence}

Jason S. Hauptman: Seattle Children's Hospital, Seattle, WA. jason.hauptman@seattlechildrens.org. 